\title{
استفدام نماذج الاستبدال في تحديد الزمن الامثل للاستبدال
}

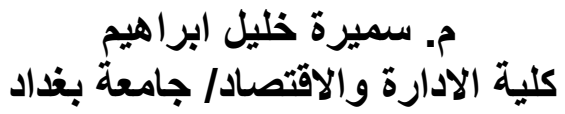 \\ قسم الاحصاء
}

\begin{abstract}
|المستصن

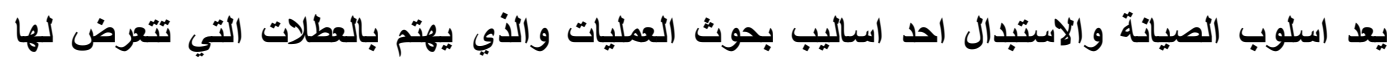

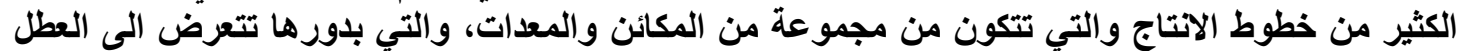

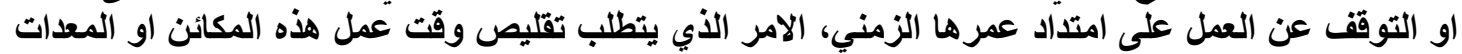

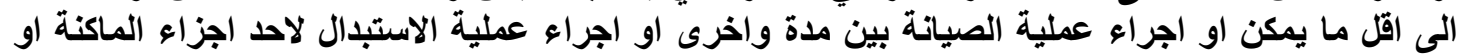

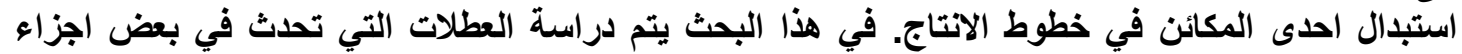

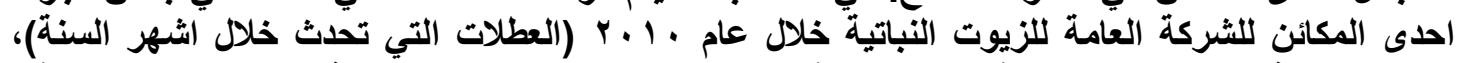

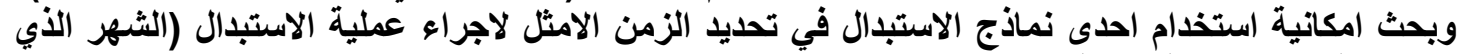

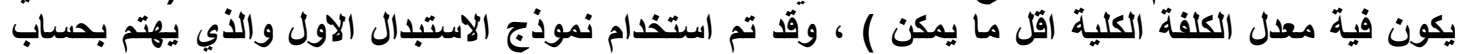

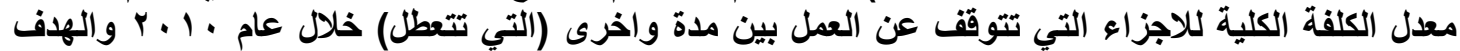

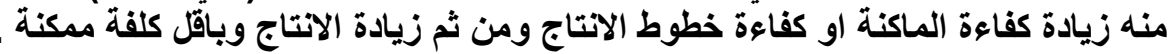

الإسطمات الاساسية :- نماذج الاستبدال - العطلات

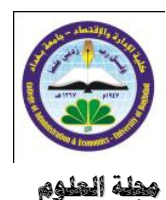

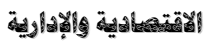

19

Mr ased

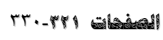




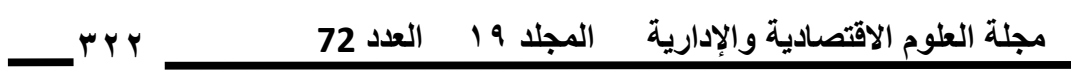 \\ 1استخدام نـماذج الاستبدال في تصديد الزمن 1الامثل للاستبدال}

يعتبر اسلوب الصيانة والاستبدال احد اساليب بحوث العمليات ، حيث يهتم هذا الاسلوب بمشاكل

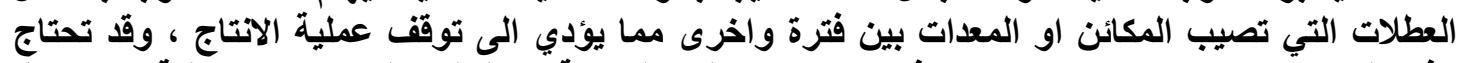

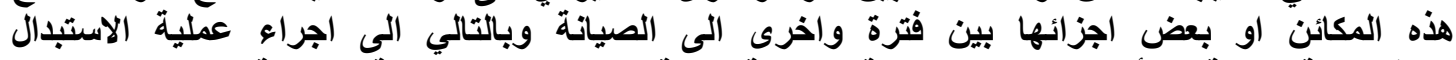

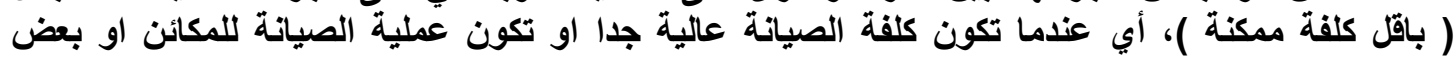

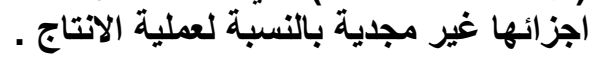

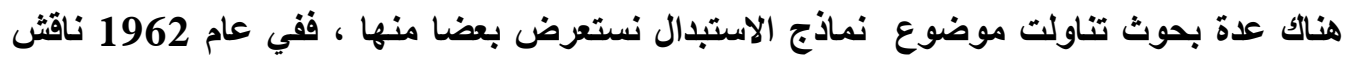

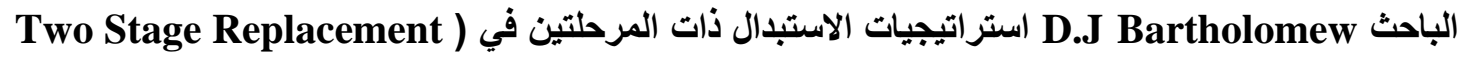

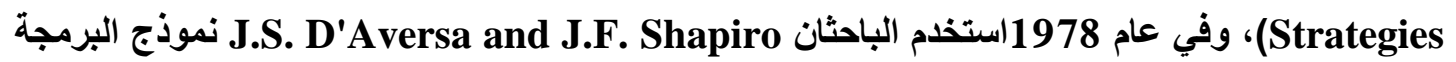
الخطية في الوصول للامثلية بالنسبة للمكائن في ( M.I. Khalil Optimal Machine Maintenance and Enumeration عام 1999 في مشاكل البرمجة الخطية العدية وتطبيقاتها في نماذج الاستبدال البال ، Programming Problem and their Application in Vehicle Replacement Models) كما ناقش الباحث نفسه في عام 2005 البرمجة العددية الضبابية المتعددة الاهداف ونماذج الاستبدال من خلال (On Vehicle Replacement Models Using Large Scale Multi-Objective Fuzzy البرو (Integer Programming)

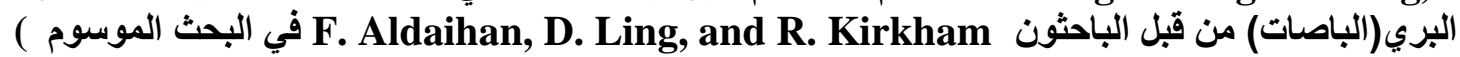
Binary Integer Algorithm for Solving Capital Assets Replacement Problem )

في هذا البحث سوف يتم تحديد الزمن الامثل لاجراء الاستبدال حيث يتم اخذ العطلات التي تحدث لبعض اجزاء احدى المكائن خلال عام 2010 ( بالاشهر) وباقّل كلفة ممكنة.

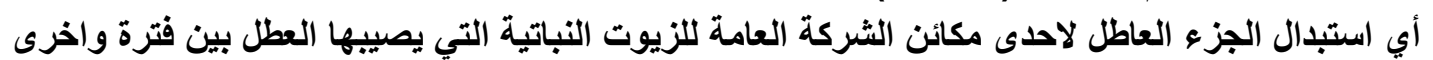

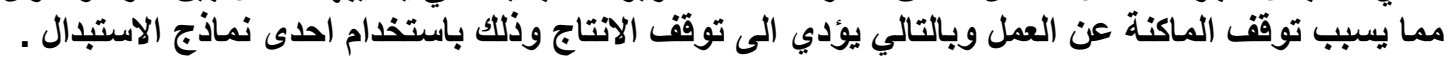

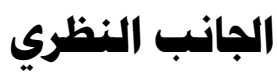

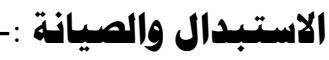

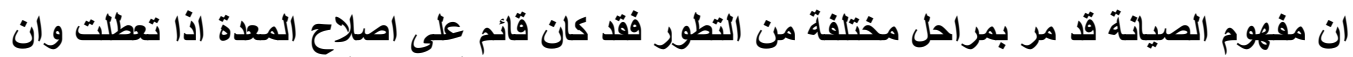

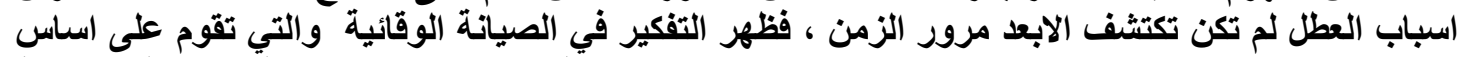

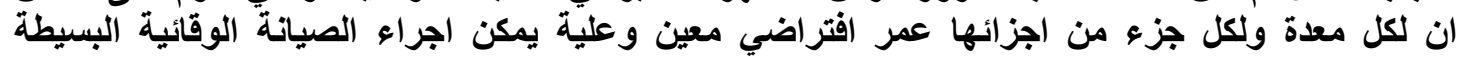

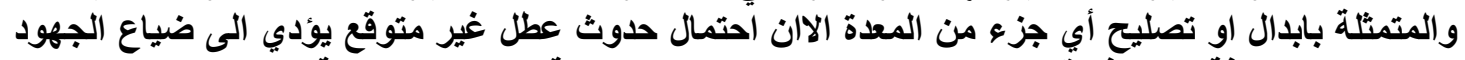

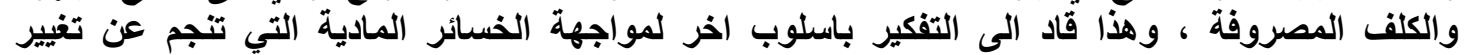

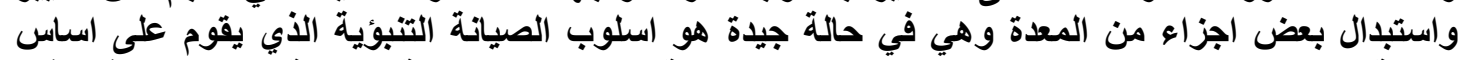

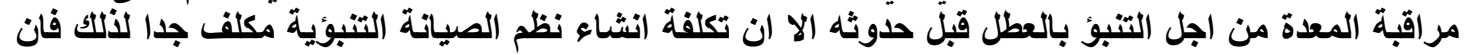

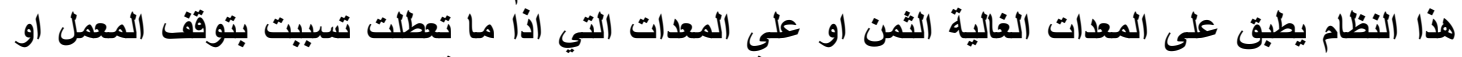

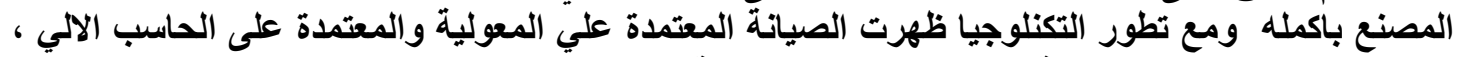

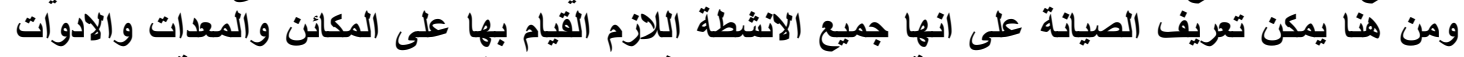

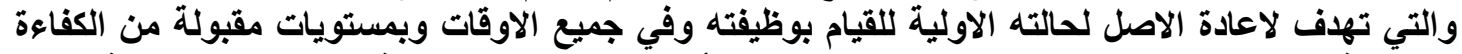

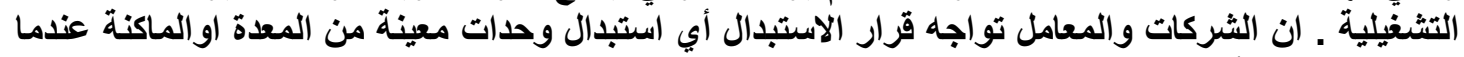

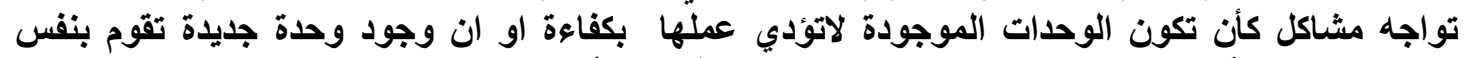

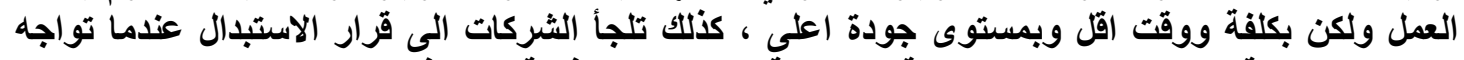

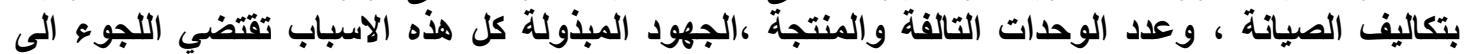

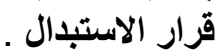




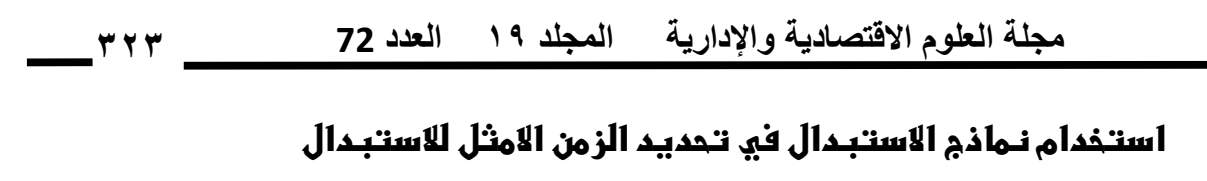

نهاذج الصيانة والاستبدال :-

في نموذج الاستبدال الاول تكون كلف الصياتة والتصليح تزداد مع الزمن حبث ان ان اتخاذ القرار اللازم

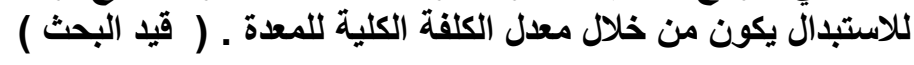

$$
\begin{aligned}
& \mathrm{TC}=\mathrm{C}+\int_{0}^{\mathrm{n}} \mathbf{f}(\mathbf{t}) \mathrm{dt}-\mathrm{s} \\
& \text { : TC : مجموع الكلف الكلية . }
\end{aligned}
$$

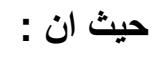

$$
\begin{aligned}
& \text { : كلفة شراء المعدة الجديدة. : C }
\end{aligned}
$$

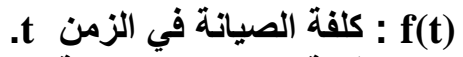

$$
\begin{aligned}
& \text { : قيمة بيع المعدة القديمة : S } \\
& \text { : n : الفترة الزمنية لخدمة المعدة . } \\
& \text { اما معدل الكلقة الكلية T فهي : }
\end{aligned}
$$$$
T=T C / n=1 / n\left\{C-S+\int_{0}^{n} f(t) d t\right\}
$$$$
\text { وان اقل معدل كلقة كلية يتحقق عندما : }
$$$$
g(n)=1 / n\left\{C-S+\int_{0}^{n} f(t) d t\right\}
$$

$$
g(n)=1 / n\left\{C-S+\sum_{t=0}^{n} f(t)\right\}
$$

t

$$
\text { t }
$$

اما في نموذج الاستبدال الثاني فيتم الاستبدال للاجزاء التي تعطلت بثنكل مفاجئ ويتم اللجوء التي سياسة الاستبدال الفردي والجماعي لكلّ فترة زمنية يتم تحديد السياستة المثلى من خلال اختيار القل معدل كلفة كلية.

$$
\begin{array}{r}
\mathbf{C I R}=\mathbf{C}_{1} * \mathbf{A F} \\
\mathbf{A F}=\mathbf{N}_{0} / \mathbf{A L} \\
\mathbf{A L}=\sum_{\mathrm{i}=1}^{\mathrm{n}} \mathbf{i P i}
\end{array}
$$

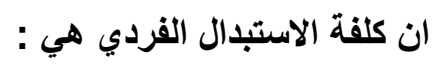

. معدل العطل في الفترة الزمنية AF

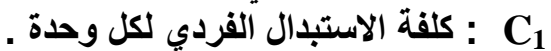

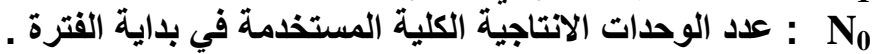

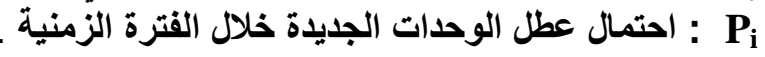
$i=1,2,3, \ldots . . ., n$ 


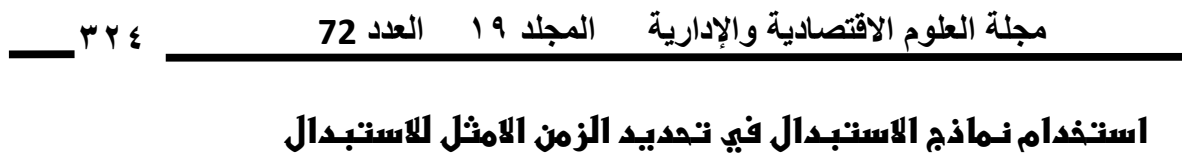

$$
\begin{aligned}
& \text { وان عدد الوحدات المستبدلة خلال الفترة i هي : } \\
& N_{i}=N_{0} P i+N_{1} P_{i-1}+\ldots \ldots . .+N_{i-1} P_{1} \\
& \text { ولحساب كلفة الاستبدال الجماعي } \\
& \operatorname{ACGR}_{i}=\left\{C_{2} * N_{0}+C_{1} * \sum_{j=1}^{i} N j\right\} / i \\
& \text { حيث ان : } \\
& \text { كلفة الاستبدال الجماعي لكل وحدة . Cي }
\end{aligned}
$$

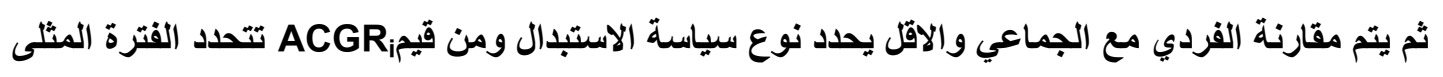
املانتبوذج الصيانة فيستخدم عندما يكون من الممكن اصلاح المعدة وفي هذه النماذج يستخدم الوقت المتوقع

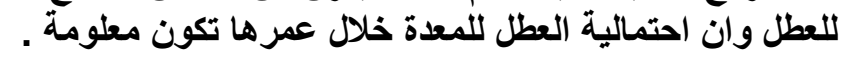
$\mathbf{C M}=\{\mathrm{MC} / \mathrm{EL}\} * \mathbf{N}_{\mathbf{0}}$

حيث ان :

: CM : Cلقة الصيانة : : : كلف التصليح لكل وحدة : CMC

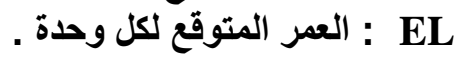

\section{البانب التطبيقي :-}

تعتبر الثركة العامة للزيوت النباتية من اكبر الثركات في العراق حيث تلعب دورا مهما في دعم

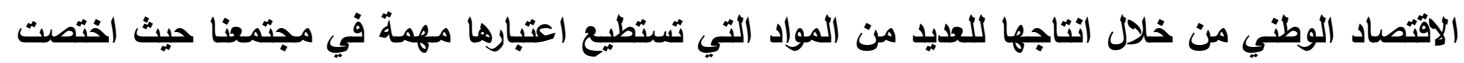
في صناعة الزيوت النباتية السائلة، والسمن النباتي، والصوابين، ومستحضرات التجميل ، ومساحيق الغسيل .

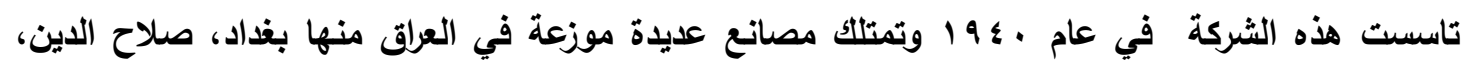
ميسان . كل مصنع يحتوي على مجموعة من خطوط الانتاج وكل خط انتاج يحتوي على مجموعة من المكائن

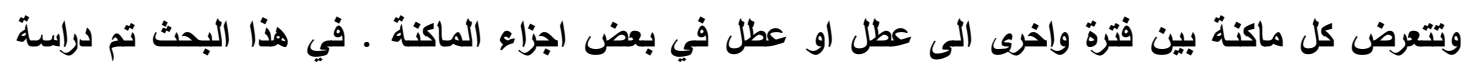
العطلات التي تحث في بعض اجزاء احدى المكائن ( - shaft complete,role rubber, spindle, die

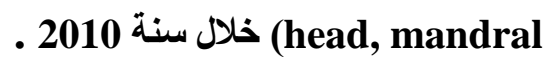


استخدام نـماذج 1استبدال في تصديد الزمن الامثل للاستبدال

\begin{tabular}{|c|c|c|c|}
\hline كلفة تصليح العطل(الف دينار) & عدد العطلات & وقت العطل (الثهر) & $ت$ \\
\hline 57 & 1 & كانون الثاني & 1 \\
\hline 114 & 1 & شباط & $r$ \\
\hline ---- & $\begin{array}{ll}--- \\
---\end{array}$ & اذار & $r$ \\
\hline 171 & 1 & نيسان & $\varepsilon$ \\
\hline ---- & ---- & ايار & 0 \\
\hline 228 & 1 & حزيران & 7 \\
\hline---- & ---- & تموز & V \\
\hline ----- & $\begin{array}{ll}--- \\
--\end{array}$ & اب اب اب & $\Lambda$ \\
\hline 285 & 1 & ايلول & 9 \\
\hline ---- & ---- & تشرين الاول & 1. \\
\hline 342 & 1 & تثرين الثاني & 11 \\
\hline 399 & 1 & كانون الاول & IT \\
\hline
\end{tabular}

جدول رقم (1) يبين اوقات العطلات وكلف تصليح العطل ــ mandral

\begin{tabular}{|c|c|c|c|}
\hline كلفة تصليح العطل (الف دينار) & عدد العطلات & وقت العطل (الثهر) & ت \\
\hline 25 & 1 & كانون الثاني & 1 \\
\hline 50 & 1 & شباط & $r$ \\
\hline 100 & 2 & اذار & $r$ \\
\hline 150 & 2 & نيسان & $\varepsilon$ \\
\hline 200 & 2 & ايار & 0 \\
\hline 275 & 3 & حزيران & 7 \\
\hline 325 & 2 & تموز & V \\
\hline 400 & 3 & اب & $\Lambda$ \\
\hline 450 & 2 & ايلول & 9 \\
\hline 500 & 2 & تشرين اول & 1. \\
\hline 550 & 2 & تشرين ثُاني & 11 \\
\hline 600 & 2 & كانون الاول & IY \\
\hline
\end{tabular}

جدول رقم (ץ) يبين وقت وعدد العطلات وكلف تصليح العطل دـ Shaft complete 


\begin{tabular}{|c|c|c|c|}
\hline كلفة تصليح العطل(الف دينار) & عدد العطلات & وقت العطل(الشهر) & $ت$ \\
\hline--- & --- & كانون الثاني & 1 \\
\hline 14 & $\mathbf{1}$ & شباط & $r$ \\
\hline 28 & 1 & اذار & $r$ \\
\hline 42 & 1 & نيسان & $\varepsilon$ \\
\hline 70 & 2 & ايار & 0 \\
\hline --- & -- & حزيران & 7 \\
\hline 84 & 1 & تموز & $\mathrm{v}$ \\
\hline$-\cdots$ & --- & اب & $\Lambda$ \\
\hline--- & --- & ايلول & 9 \\
\hline 98 & 1 & تثثرين اول & 1. \\
\hline --- & --- & تشرين ثاني & 11 \\
\hline 112 & 1 & كانون الاول & ir \\
\hline
\end{tabular}

جدول رقم ("آ) يبين وقت وعدد العطلات وكلف تصليح العطل لـ role rubber

\begin{tabular}{|c|c|c|c|}
\hline كلفة تصليح العطل(الف دينار) & عدد العطلات & وقت العطل(الثهر) & $ت$ \\
\hline 11 & 1 & كانون الثاني & 1 \\
\hline 22 & 1 & شباط & $r$ \\
\hline 33 & 1 & اذار & $r$ \\
\hline 55 & 2 & نيسان & $\varepsilon$ \\
\hline 66 & 1 & ايار & 0 \\
\hline 77 & 1 & حزيران & 7 \\
\hline 88 & 1 & تموز & $\mathrm{v}$ \\
\hline-- & -.- & اب & $\Lambda$ \\
\hline 99 & 1 & 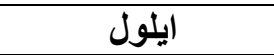 & 9 \\
\hline 110 & 1 & تثرين اول & 1. \\
\hline 121 & 1 & تثرين ثاني & 11 \\
\hline--- & -.- & كانون الاول & ir \\
\hline
\end{tabular}

جدول رقم ( ) يبين وقت وعدد العطلات وكلف تصليج العطل لـ Spendle 
استخدام نماذج الاستبدال في تصديد الزمز الامثل للاستبدال

\begin{tabular}{|c|c|c|c|}
\hline كلفة تصليح العطل(الف دينار) & عدد العطلات & وقت العطل(الثهر) & ت \\
\hline --- & --- & كانون الثاني & 1 \\
\hline 23 & 1 & شباط & $r$ \\
\hline 46 & 1 & اذار & $r$ \\
\hline--- & --- & 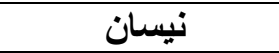 & $\varepsilon$ \\
\hline 69 & 1 & ايار & 0 \\
\hline 92 & 1 & حزيران & 7 \\
\hline 115 & 1 & تموز & $\mathrm{V}$ \\
\hline--- & --- & اب & $\Lambda$ \\
\hline 184 & 3 & ايلول & 9 \\
\hline 207 & 1 & تشرين اول & 1. \\
\hline $\begin{array}{ll}-- \\
\end{array}$ & $\begin{array}{ll}-- \\
\end{array}$ & تشرين ثُاني & 11 \\
\hline 230 & 1 & كانون الاول & $1 T$ \\
\hline
\end{tabular}

جدول رقم (0) يبين وقت و عدد العطلات وكلف تصليح العطل د D Die-Head

وكان سعر الثراء من السوق لهذه الاجزاء كما هو مبين في الجدول ادناه :-

\begin{tabular}{|c|c|c|}
\hline سعر الثراء (الف دينار) & الجزء العاطل & ت \\
\hline 360 & mandral & 1 \\
\hline 775 & shaft complete & $r$ \\
\hline 75 & role rubber & $r$ \\
\hline 360 & spendle & $\varepsilon$ \\
\hline 200 & Die-Head & 0 \\
\hline
\end{tabular}

جدول رقم (†) يبين سعر الثراء 


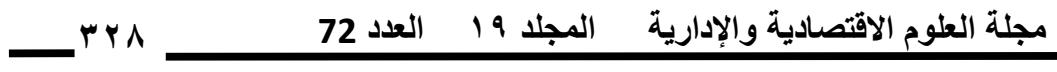 \\ استخدام نـماذج 1لاستبدال في تصديد الزمن الامثل للاستبدال}

وعند استخدام النموذج الاول من نماذج الاستبدال وتطبيق المعادلة (2) كاتت النتائج كالاتي

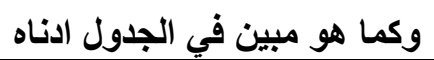

\begin{tabular}{|c|c|c|c|c|c|c|}
\hline \multicolumn{5}{|c|}{ 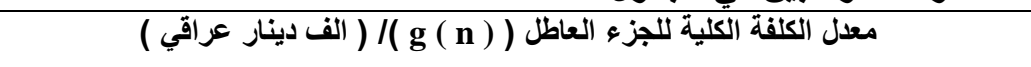 } & \multirow{2}{*}{ الجزء العاطل } & \multirow[t]{2}{*}{$ت$} \\
\hline Die-Head & spendle & Role rubber & $\begin{array}{c}\text { shaft } \\
\text { complete }\end{array}$ & Mandral & & \\
\hline--- & 387 & ---- & 800 & 417 & كانون الثاتى & 1 \\
\hline 111.5 & 220.5 & 46.5 & 425 & 265.5 & شباط & $r$ \\
\hline 89.6 & 174 & 43 & 316.6 & --- & اذار & $r$ \\
\hline--- & 164.25 & 37.75 & 275 & 175.5 & نيسان & $\xi$ \\
\hline 67.6 & 158.4 & 54.6 & 260 & +--- & ايار & 0 \\
\hline 71.6 & 223.5 & --- & 262.5 & 155 & حزيران & 7 \\
\hline 77.8 & 222.4 & 54.4 & 271 & --- & تموز & $\mathrm{v}$ \\
\hline--- & 225 & --- & 287.5 & --- & اب & $\Lambda$ \\
\hline 81 & 230 & --- & 305.5 & 135 & ايلول & 9 \\
\hline 103.6 & --.- & 39.9 & 325 & --- & تثرين الاول & 1. \\
\hline +--- & 236.7 & --- & 345.4 & 141.5 & تثرين الثاني & 11 \\
\hline 105.5 & --- & 45.2 & 366.6 & 153 & كانون الاول & ir \\
\hline 67.6 & 158.4 & 37.75 & 260 & 135 & \multicolumn{2}{|c|}{ اقل معدل كلفة كلية (الف دينار) } \\
\hline ايار & ايار & نيسمان & ايار & ايلول & \multicolumn{2}{|c|}{ الزمن الامثل للاستبدال(شهر) } \\
\hline
\end{tabular}

جدول رقم (V) يبين اقل معدل كلفة كلية والزمن الامثل للاستبدال لبعض اجزاء احدى المكائن 
ان استبدال الاجزاء العاطلة في المكائن في الوقت المناسب (اقل كلفة ) يجنب الثركة التكلفة الكبيرة

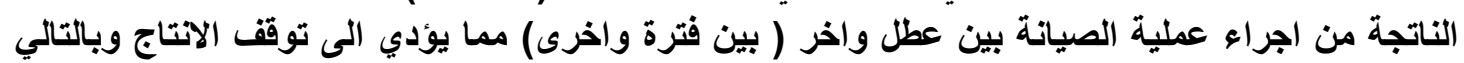

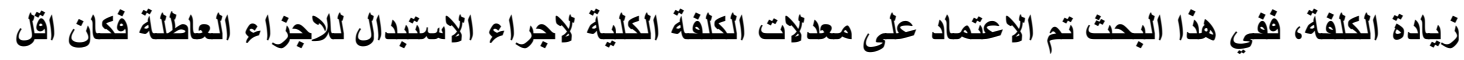
معدل للكلفة الكلية بالنسبة لـ (mandral) 135 الف دينار عراقي وكان افضل وقت للاستبدال هو في شهر ايلول، امـا ( shaft complete فقد كان من الافضل الاستبدال في شهر ايار حيث كان معدل الكلقة الكلية

هي 260 الف دينار عراقي ( الاقل ) وبنفس الاسلوب تم التعامل مع بقية الاجزاء العاطلة (Die-Head) (Role rubber ، spindle ) وقد تم الاستبدال في ( ايار،ايار، نيسان ) حيث كانت معدلات الكلفة الكلية لهذه الاجزاء هي ( 67.6 , 158.4 , 37.75) الف دينار عراقي على التوالي.

استخدام نماذج الاستبدال المناسبة لبقية اجزاء المكائن او لكافة خطوط الانتاج التي يصيبها العطل بين

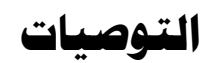
فترة واخرى لتفادي مشكلة توقف المكائن وخطوط الانتاج عن العمل مما يؤدي الى توقف العن عملية الاتتاج .

\section{|}

1.Prem Kumar Gupta and D.S. Hira " Operations Research : an introduction " $2^{\text {nd }}$ edition (1989) S. Chand \& Company LTD, NewDelhi.

2.M.I. Khalil, "In Integer Programming Problem and their Application in Vehicle ,1999.

Replacement Model", M.Sc.Thesis,Helwan University,Cairo,Egypttd hgl;hzk

3 . M.I. Khalil, " On Vehicle Replacement Models Using Large Scale Multi Objective Fuzzy Integer Programming", ph.D. Thesis,Helwan University, Cairo,Egypt,2005.

4.Hamd A. Taha, " Operations Research An Introduction " New York, $9^{\text {th }}$ Edition, 2007.

5. F. Aldaihani1, D. Ling, and R. Kirkham, " Binary Integer Algorithm for Solving Capital Assets Replacement Problem" School of Mechanical, Aerospace, and Civil Engineering, University of Manchester, UK ,2010. 


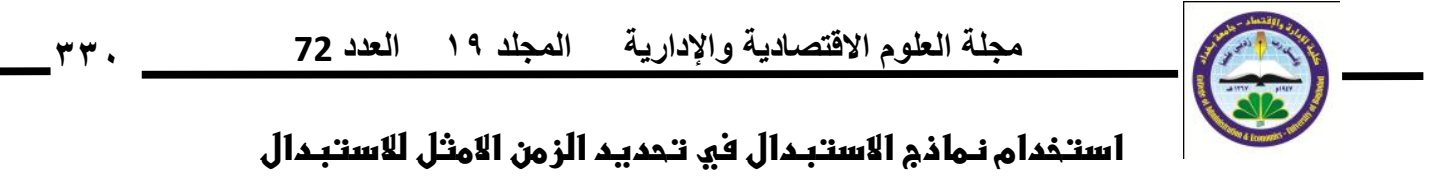

The Use of Replacement Models On Determine the Optimal Time to Replacement

\begin{abstract}
:-
The approach maintenance and replacement one of techniques of operations research whom cares of the failure experienced by a lot of production lines which consist of a set of machines and equipment, which in turn exposed to the failure or work stoppages over the lifetime, which requires reducing the working time of these machines or equipment below what can or conuct maintenance process once in a while or a replacement for one part of the machine or replace one of the machines in production lines. In this research is the study of the failure $s$ that occur in some parts of one of the machines for the General Company for Vegetable Oils in 2010 (the failure the failure $s$ that occur during the months of the year), and discuss the possibility of using one of the models replacement in determining time optimization for the replacement process (the month in which the rate total cost less what can be), I have been using a model replacement first and who cares calculate the average total cost of the parts that stop working once in a while (which break down) in 2010 and aimed at increasing the efficiency of the machine or the efficiency of production lines and thus increase production and the lowest possible cost.
\end{abstract}

Keyword : Replacement models, Failure 